\title{
Indirect benefits are a crucial consideration when evaluating SARS-CoV-2 vaccine candidates
}

To the Editor - Public-health interventions aimed at curbing the spread of the coronavirus SARS-CoV-2 have been limited to non-pharmaceutical interventions, including travel restrictions, isolation, contact tracing and quarantine, mask wearing and social distancing, with variable success worldwide. These interventions have been critical to slowing the spread of the virus, but given their substantial societal, economic and political costs, alternative long-term solutions are needed. A vaccine remains the most promising one. Thanks to tremendous worldwide research efforts, vaccine development is well underway, with more than 50 novel vaccine candidates already in clinical trials.

Clinical trials evaluate the safety and efficacy of vaccine candidates. Licensed vaccines must provide a direct protective benefit to the vaccinee. The endpoint for SARS-CoV-2 vaccine trials is the prevention of COVID-19 disease. In addition to directly protecting a proportion of vaccinated people, vaccination programs can also indirectly reduce infection risk to all susceptible people ${ }^{1}$, either by reducing the number of infected people in the population or by rendering breakthrough cases less infectious. Vaccinated people who later become infected may have less viral shedding, fewer symptoms or faster recovery time, all of which could reduce the risk of transmission to an uninfected person.

Quantifying the indirect effects of vaccination on population-level outcomes such as disease incidence and mortality usually requires evaluation after a vaccine has been in use for some time $^{2}$. However, mathematical modeling can help researchers bridge this gap and assess population-level effects in advance in order to gauge whether a vaccine should be further considered, even if the direct protective effects are lower than desired. For example, modeling of vaccine-allocation strategies for pandemic influenza showed that increases in vaccine efficacy against infection support increased prioritization of vaccinating people in age groups other than those at greatest risk of mortality ${ }^{3}$. With a similar model, we have demonstrated that a vaccine that modestly reduces the risk of clinical disease, but greatly decreases infectiousness, can be more beneficial at the population level than a vaccine that prevents most symptomatic SARS-CoV-2 infections, but does little to reduce onward transmission ${ }^{4}$. Strategic monitoring and targeted data collection during clinical trials, and perhaps challenge studies, can improve model parameterization by providing relevant information about the effect of vaccination on the potential for onward transmission. Specifically, susceptibility to symptomatic infection versus asymptomatic infection, peak viral load and degree of viral shedding, as well as infection duration, can all be measured. Independently, such data do not suffice to predict the indirect effects of vaccines at the population level, but they can be incorporated into epidemiological models that can forecast possible outcomes.

The main drawbacks to collecting such data are the increase in time and resources required and the challenge of frequently testing and monitoring large numbers of participants. These data might be more easily collected in challenge studies than in clinical trials: fewer participants are required, and since the challenge time is known and the inoculum dose is controlled, measurements can be taken more reliably and differences between study arms may be more readily apparent. But in the absence of an effective treatment for COVID-19, human challenge studies assessing vaccine efficacy remain unlikely to be approved for ethical reasons, although some argue that the benefits can outweigh the costs 5 . The risk of serious adverse effects or death following experimental infection is typically deemed unacceptable for a person who would not otherwise be exposed. Challenge studies in animal models offer an alternative, despite likely considerable differences between humans and non-human primates in viral dynamics and infection. Two vaccine challenge studies in rhesus macaques have already shown that SARS-CoV-2 vaccines can be moderately successful in preventing disease $^{6,7}$. In one of these studies, the vaccine candidate AZD1222 reduced viral load, which can reduce transmission potential; however, the vaccine did not reduce nasal shedding or eliminate infectious virus in all animals, which suggests that the potential for transmission may not be entirely averted by vaccination ${ }^{6}$.
We argue that in parallel to assessing the efficacy and safety of vaccine candidates for individual recipients, research aimed at gauging indirect benefits is a critical area of investigation. In assessing vaccine candidates, it should be recognized that a vaccine can have a moderate primary failure rate but nonetheless serve to reduce infectiousness and onward transmission. The indirect effects of SARS-CoV-2 vaccines may be particularly crucial given the severity profile of COVID-19. The risk of hospitalization and mortality increases substantially with age, but vaccination of older people is often less effective due to immunosenescence. Furthermore, because of indirect effects, overall morbidity and mortality can be reduced even when vaccination coverage is modest, if the right groups are targeted ${ }^{8}$. This is a crucial consideration, given that extensive coverage will be a challenge due to insufficient initial supply, delays in infrastructure delivery scale-up, and vaccine hesitancy. Therefore, indirect effects are critically important to consider when evaluating SARS-CoV-2 vaccine candidates and formulating strategies for their rollout.

\section{Molly E. Gallagher(D) ${ }^{凶}$, Andrew J. Sieben², Kristin N. Nelson ${ }^{3}$, Alicia N. M. Kraay ${ }^{3}$, Walter A. Orenstein ${ }^{4,5}$, Ben Lopman ${ }^{3}$, Andreas Handel ${ }^{6}$ and Katia Koelle ${ }^{1,5}$ ${ }^{1}$ Department of Biology, Emory University, Atlanta, GA, USA. ${ }^{2}$ School of Medicine, Emory University, Atlanta, GA, USA. 'Department of Epidemiology, Rollins School of Public Health, Emory University, Atlanta, GA, USA. ${ }^{4}$ Emory Vaccine Center, School of Medicine, Emory University, Atlanta, GA, USA. ${ }^{5}$ Emory-UGA Center of Excellence of Influenza Research and Surveillance, Atlanta, GA, USA. ${ }^{6}$ Department of Epidemiology and Biostatistics, College of Public Health, University of Georgia, Athens, GA, USA.

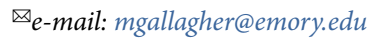

Published online: 23 November 2020 https://doi.org/10.1038/s41591-020-01172-x

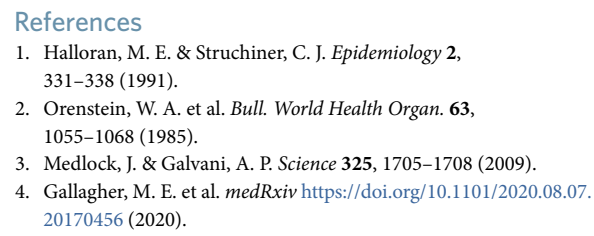


5. Eyal, N., Lipsitch, M. \& Smith, P. G. J. Infect. Dis. 221, 1752-1756 (2020).

6. van Doremalen, N. et al. Nature 586, 578-582 (2020).

7. Yu, J. et al. Science 369, 806-811 (2020).

8. Eichner, M., Schwehm, M., Eichner, L. \& Gerlier, L. BMC Infect. Dis. 17, 308 (2017).
Acknowledgements

The research reported in this paper was supported by US National Institutes of Health National Institute of General Medical Sciences grant 1R01 GM12428003S1 (supplement); National Science Foundation grant 2032084; and National Institute of Allergy and
Infectious Diseases Centers of Excellence for Influenza Research and Surveillance (CEIRS) grant HHSN272201400004C.

Competing interests

The authors declare no competing interests.

\section{Toward superhuman SARS-CoV-2 immunity?}

To the Editor - If asked, many scientists would probably agree with the statement 'Natural infection gives better immunity than vaccination'. Indeed, if one survives the infection, there are certainly many pathogens for which natural infection induces stronger immune responses and more long-lived immunity than does vaccination. Measles is prototypic of this ${ }^{1}$. While there was a clear risk, after infection, of death, encephalitis and pneumonia before there was a vaccine, survivors gained lifelong immunity. Vaccination against measles, on the other hand, requires two shots and may not offer lifelong complete protection but has proven to be good enough to keep the disease in check when widely implemented.

In contrast to the measles virus, there are a number of pathogens for which vaccination generates stronger immune responses and more-effective protection against disease than does natural infection. In these cases, the man-made vaccine is 'superhuman'; that is, it gives humans immune responses superior to those generated in response to infection. The bacterium that causes tetanus is a notable example of this. Infection with this pathogen results in production of the highly potent tetanus toxin in small amounts that are sufficient to cause severe disease but not enough to generate a strong immune, particularly antibody, response. On the other hand, vaccination with an inactivated form of the toxin (tetanus toxoid) generates antibody responses sufficient to provide protection against the toxin for a decade and probably longer ${ }^{2}$. Hence, vaccination is recommended even for those who have been infected with the bacterium that causes tetanus and have shown clinical symptoms, as well as those who have been merely potentially exposed.

Another example from the bacterial world is Haemophilus influenza type b (Hib). Hib causes a variety of serious conditions, including meningitis, pneumonia and septicemia. The surface of the bacterium is protected by a coating of sugars, which typically induce rather poor antibody responses. However, the responses can be greatly enhanced by linkage of the sugars to a protein in a vaccine in preparations known as 'glycoconjugates'3. The typical responses to vaccination are therefore greatly enhanced relative to the responses to natural infection. The vaccine is now given to children under the age of 2 years in many developed countries in particular and has greatly reduced the incidence of meningitis due to Hib.

Among viruses, two classic cases in which vaccines generate immunity superior to that generated by natural infection are varicella zoster virus, which can lead to shingles, and human papillomavirus (HPV), some strains of which cause various malignancies, including cervical, penile and oropharyngeal cancer. Varicella zoster virus typically causes chickenpox in children and young adults and is resolved but rendered latent so that when re-activated in later life, it can lead to shingles. Immunity arising from the primary infection does not prevent the disease in those who develop shingles. However, the recently developed vaccines Zostavax and Shingrix do offer protection against shingles. Shingrix protects around $90 \%$ vaccinees across all age groups, and it is suggested for an extended time period ${ }^{4}$. Protection seems to be antibody based but with important contributions from $\mathrm{CD} 4^{+} \mathrm{T}$ cells.

The quintessential example of immunity superior to that induced by infection is the vaccine against HPV. The HPV strains that cause genital cancers enter the body via genital mucosal surfaces, and the antibody responses induced are low and take a long time to develop-more than 8 months in one study ${ }^{5}$. In contrast, two or three sequential intramuscular injections of one of the vaccines against HPV induce potent neutralizing antibody responses that have been shown directly in an animal model to prevent entry of the virus into target cells and the establishment of infection ${ }^{6}$. The vaccines against HPV are based on the incorporation of a single viral surface protein into virus-like particles. They have been shown to offer complete protection against cervical cancer.

Where does the coronavirus SARS-CoV-2 lie along the spectrum of natural infection versus vaccine-induced protective efficacy? The answer to this question will be known only as more data are collected from ongoing natural infection and vaccine studies; the initial results from interim analyses by Pfizer/ BioNTech and Moderna of mRNA vaccines against SAR-CoV-2 showing a reduction in infections of around $95 \%$ are very encouraging ${ }^{7}$. There are a number of other promising signs for vaccines. Protection against infection and disease has been associated with neutralizing antibodies in both vaccine studies and passive-antibody-transfer studies in animal models ${ }^{8}$. Furthermore, passive antibodies seem to have beneficial effects on established early SARS-CoV-2 infection in humans, which suggests that they can contribute to protection ${ }^{9}$. Many of the current vaccines in clinical trials ${ }^{10}$ induce high levels of neutralizing antibodies that animal model studies predict would provide protection. Furthermore, even if the levels reached do not provide complete sterilizing immunity and are insufficient to prevent the upper-respiratory-tract symptoms typical of the common cold, they may prevent serious lower-respiratory-tract disease. The disadvantage of such an outcome is that the vaccine probably would not prevent ongoing transmission from an infected vaccinee. In contrast to many of the vaccines, natural infection induces highly variable levels of neutralizing antibodies, a proportion of which may not provide immunity. At the patient level, there are isolated reports of re-infection with SARS-CoV-2 associated with an insufficient initial antibody response. A second likely contributor to protection against SARS-CoV-2 is cellular immunity ${ }^{11}$, although the data on its importance are not yet clear. A number of vaccines are expected to induce substantial cellular immune responses. One important unknown factor in the context of both natural infection and vaccination is the durability of immune responses. Multiple longitudinal cohort studies of antibody levels after COVID-19 have shown that they are variable, with some showing durability over several months and others showing some 'fall-off'. The durability of antibody 